\title{
La fonction nerveuse périphérique et la souffrance liée aux symptômes pendant la biothérapie du mélanome malin
}

\author{
par Constance G. Visovsky
}

\section{Abrégé}

Les études sur les changements physiologiques induits par la biothérapie sont peu nombreuses, et le suivi systématique des effets neurotoxiques indésirables, insuffisant. Le but de cette étude pilote était de déterminer l'altération de la fonction nerveuse périphérique et l'évolution de la souffrance liée aux symptômes pendant le traitement par biothérapie du mélanome malin. On a mesuré la fonction nerveuse périphérique au niveau de référence et à 4 et 12 semaines de traitement à l'interféron alpha, auprès d'un échantillon de convenance de 11 participants atteints d'un mélanome malin. Les données ont été analysées à l'aide des tracés et des courbes de régression, afin de déterminer les changements survenus au niveau du toucher, de la marchelde l'équilibre, de la vision, de l'audition, de la sensibilité vibratoire, de la force musculaire, des réflexes tendineux, de la tension artérielle et de la souffrance liée aux symptômes. Des dégradations de l'audition, du toucher, de la sensibilité vibratoire et de la force musculaire ont été observées, ainsi que des modifications de l'acuité visuelle et de la tension artérielle orthostatique, tandis que la marchell'équilibre restaient stables. En outre, des symptômes neuropathiques étaient associés à la souffrance liée aux symptômes. La caractérisation de tels changements peut rehausser notre compréhension de la nature des effets physiologiques de la biothérapie à haute dose et aider les cliniciens à mieux préparer les patients aux changements fonctionnels auxquels ils doivent s'attendre ainsi qu'aux adaptations ultérieures de leur mode de vie. Dans le cadre d'une étude plus large de ce phénomène, ces conclusions pourraient constituer une source d'information sur des résultats importants et sur les intervalles des mesures de la neuropathie induite par la thérapie et sur l'allègement de la souffrance liée aux symptômes chez les patients recevant un traitement du cancer à base d'agents biologiques.

Mots clés: neuropathie périphérique, biothérapie, clinique, mesure

\section{Introduction}

On estime que 54000 nouveaux cas de mélanome malin seront diagnostiqués chaque année, dont plus de 7000 connaîtront une issue fatale (American Cancer Society, 2003). L'Institut national du cancer du Canada estimait qu'il y aurait, en 2004, 4200 nouveaux cas de mélanome, entraînant 810 décès (Statistiques canadiennes sur le cancer, 2004). Le traitement du mélanome malin implique souvent l'usage d'agents biologiques, en particulier l'interféron alpha-2b. Mais si ces agents peuvent prolonger la vie, ils ont aussi des effets secondaires importants. Des preuves cliniques toujours plus abondantes révèlent que le traitement du cancer au moyen d'agents biologiques provoque un certain degré de neuropathie périphérique (Irwin, 1987; Smith et al., 1991; Vial et Descotes, 1994; Merriggioli et Brown, 2000). La neuropathie périphérique est une dégradation des neurones périphériques, moteurs, sensoriels et autonomes qui prédispose le sujet à des blessures. Les déficits de neurones moteurs entraînent une déperdition musculaire et une faiblesse dans les extrémités inférieures. Les lésions des neurones sensoriels se traduisent par des pertes de sensibilité dans les mains et les pieds - les fameuses « anesthésie en chaussette » et « anesthésie en gant ». Les manifestations cliniques de la neuropathie périphérique comprennent la perte de sensibilité tactile, une perception altérée de la température, la faiblesse, la douleur, l'engourdissement et les sensations de fourmillement. L'examen physique révèle, entre autres, une diminution ou la perte des réflexes tendineux et une détérioration de la sensibilité vibratoire. Les lésions associées des nerfs crâniens provoquent une baisse de l'audition et de la perception visuelle (Visovsky, 2003).

Une relation a été établie entre les agents biologiques tels que l'interféron alpha et une neuropathie axonale à prédominance sensorielle qui apparaît dans les semaines suivant le début de la thérapie et persiste pendant plusieurs mois au-delà de l'arrêt du traitement (Fatovich et al, 1996; Gastineau, Habermann et Hermann, 1989; Manesis, Petrou, Brouzas et al., 1994; Quattrinin, Comi, Nemmmni, Martinelli, Villa, Caimi, Wrabetz et Canal, 1997). On sait que la neuropathie consécutive à l'administration d'interféron alpha est cumulative et qu'elle dépend des doses employées (Armstrong, Rust et Kohtz, 1997; Berger et al., 1997; Meyers, Scheibel et Forman, 1991; Gastineau et Habermann, 1989; Gotaskie et Andreassi, 1994; Wilkes, 1996).

Provenant pour la plupart d'études de cas, les informations sur la neuropathie induite par la biothérapie sont limitées de par la nature rétrospective indirecte des données utilisées et par l'absence de liens entre la neuropathie et des différences mesurables dans les marqueurs physiologiques. Les sujets qui reçoivent des agents biologiques pour un mélanome malin n'ont pas fait l'objet d'une surveillance systématique des effets à long terme ni de la souffrance liée aux symptômes, en dépit du fait que la neuropathie périphérique est responsable de désordres fonctionnels auxquels les patients et leur famille sont mal préparés. Cette étude pilote a donc examiné l'évolution de la fonction nerveuse périphérique chez les sujets soumis à un traitement biothérapique du mélanome malin. En particulier, l'étude a observé les modifications caractéristiques de la fonction nerveuse périphérique (vision, audition, sensibilité vibratoire, réflexes tendineux, sensibilité cutanée, marche, équilibre, force musculaire, tension artérielle orthostatique) et examiné les relations entre les symptômes de neuropathie périphérique éprouvés et la souffrance liée aux symptômes sur 12 semaines de traitement.

\section{Méthodes}

Les données ont été collectées avant le commencement de la thérapie (niveau de référence), et à 4 et à 12 semaines de traitement. Les mesures prises au niveau de référence avaient pour but de dégager les baisses préexistantes de la fonction nerveuse périphérique, sans rapport avec le déroulement de la chimiothérapie. Le point de mesure de la 4e semaine a été choisi pour coïncider avec la fin d'un mois de thérapie d'induction à haute dose, avec le point de mesure restant à 12 semaines pour évaluer la fonction nerveuse périphérique à des doses standard plus faibles.

\section{Cadre}

Cette étude pilote a été menée dans un centre anticancéreux agréé par l'Institut national du cancer des États Unis et dans un centre de cancérologie ambulatoire communautaire situé dans la région du Middle West. L'échantillon était composé de 11 adultes ayant fait l'objet d'un

Constance G. Visovsky, inf., PhD, ACNP, est professeure agrégée, University of Nebraska Medical Center, College of Nursing, Omaha, Nebraska. Veuillez addresser vos questions à Dani Wilson-Baxteràdwilsonb@unmc.edu

Cette étude a été financée par une subvention de l'Oncology Nursing Society Foundation 
diagnostic histologique confirmé de mélanome malin, et dont le plan de traitement comportait l'administration d'interféron alpha-2b. Aucun des participants n'avait suivi de traitement de chimiothérapie ou de biothérapie auparavant, et ne présentait aucune maladie ni aucun trouble affectant la fonction nerveuse périphérique. L'échantillon était constitué de 7 femmes $(63,6 \%)$ et de 4 hommes $(36,4 \%)$, âgés de 28 à 74 ans, avec une moyenne d'âge de 53 ans (écart-type $=18,9$ ). Tous les sujets de cette étude étaient de race blanche. Le stade I de la maladie a été observé chez un participant $(9,1 \%), 4(36,4 \%)$ participants présentaient le stade II de la maladie, 4 participants $(36,4 \%)$ étaient atteints de la maladie au stade III, et $2(18,2 \%)$ avaient été diagnostiqués au stade IV. Cinq sujets avaient subi seulement une excision de leur mélanome, tandis que les 6 autres avaient subi une excision et un évidement ganglionnaire.

\section{Mesure des variables et procédures}

Après l'obtention du consentement du comité d'examen des établissements, les infirmières cliniciennes des deux sites ont communiqué la liste des sujets répondant aux critères d'admissibilité à la chercheuse qui a pris contact avec eux dans des cliniques externes, en vue de leur participation à l'étude. Avec le consentement éclairé de chaque sujet, toutes les mesures de la fonction nerveuse périphérique ont été relevées à trois reprises : au niveau de référence (avant le traitement à l'interféron alpha-2b), et à 4 et 12 semaines de traitement, respectivement. Les données démographiques avaient été collectées auparavant, au moment de l'inscription des participants. Les mesures cliniques de la fonction nerveuse périphérique concernaient l'acuité visuelle, l'acuité auditive, la sensibilité cutanée, la sensibilité vibratoire, les réflexes, la tension artérielle, la force musculaire, la marche et l'équilibre. La souffrance liée aux symptômes a été mesurée sur l'échelle de perception des symptômes (Rhodes et McDaniel, 2001), élargie pour cette étude aux 14 éléments reflétant les symptômes associés à la neuropathie périphérique. Les instruments et les cotations utilisés dans cette étude sont esquissés dans le tableau 1 et brièvement résumés cidessous.

Vision. L'acuité visuelle de chaque oeil a été testée au moyen d'une échelle Snellen manuelle. Les valeurs plus élevées reflètent une dégradation plus prononcée de l'acuité visuelle. L'échelle de lecture de Snellen fournit une mesure sensible et fiable des altérations de la vision (Bradley, Hook et Haeseker, 1991). Les résultats de chaque test de vision de près ont été exprimés sous forme de fraction pour chaque oeil, avec le dénominateur obtenu comme note, le numérateur de 20 étant constant.

Audition. L'audition a été mesurée par les tests auditifs cliniques de conduction aérienne et de conduction osseuse de Rinne et de Weber avec un diapason calibré à $250 \mathrm{~Hz}$. La fourche du diapason excité était placée contre les apophyses mastoïdes gauche et droite pour vérifier la conduction osseuse. Le diapason était ensuite dirigé vers le devant de l'oreille testée pour vérifier la conduction aérienne. Dans cette étude, pour l'épreuve de conduction aérienne de Rinne, chaque oreille était testée séparément. L'épreuve de Rinne a une sensibilité documentée de 0,84 , indépendamment du type ou de la gravité de la perte auditive (Miltenburg, 1994). Pour l'épreuve de Weber, la base du diapason vibrant était appliquée au sommet du crâne, au milieu du méridien. La note zéro signifie que le son était perçu seulement par une oreille (latéralement), un résultat anormal, et la note 1 qu'il pouvait être entendu par les deux oreilles (symétriquement), un résultat normal. Une étude utilisant l'épreuve de Weber a établi la sensibilité de ce test à détecter la perception des schémas normaux et anormaux de la conduction osseuse du son de l'appareil auditif. (Haapaniemi, Suonpaa, Salmivalli et Virolainen, 1996).

Sensibilité cutanée. Le toucher a été mesuré à l'aide des monofilaments de Semmes-Weinstein. Ces filaments de nylon calibrés plient sous l'effet d'une pression déterminée. Le filament est appliqué pendant 3 secondes sur des zones spécifiques de chaque main ( 6 zones) et de chaque pied (4 zones), pendant que le sujet garde les yeux fermés. La note obtenue correspond au monofilament ressenti, les valeurs supérieures indiquant une sensibilité moindre au toucher. Cette méthode standardisée offre un intervalle de confiance de $95 \%$ (Birke et Sims, 1985).

Sensibilité vibratoire. La sensibilité à la vibration a été testée avec un diapason de $128 \mathrm{~Hz}$, excité et appliqué sur les parties latérales droite et gauche du genou et de la cheville, et mesurée selon des procédés cliniques communément utilisés. Étant donné que les déficits vibratoires sont parmi les premières manifestations des neuropathies axonales, leur dépistage permet une détection précoce. Les seuils de perception des vibrations se sont révélés une méthode fiable et valide d'évaluation de la neuropathie périphérique, et leurs résultats recoupent tout à fait les tests de vitesse de conduction nerveuse (Klima, Weigand et DeLisa, 1991; Rendell, Katims et Richter, 1989).

Réflexes tendineux. Les réflexes tendineux ont été mesurés au genou et à la cheville avec un marteau à réflexes de Babinski, suivant un procédé clinique standardisé. Les réflexes étaient classés selon l'échelle du réflexe myotatique du National Institute of Neurological Disorders and Stroke (NINDS), graduée de 1 à 4 . Chaque réflexe était noté séparément et les notes des quatre zones testées additionnées, donnant un total qui pouvait aller de 0 (aréflexie) à 16 (hyperréflexie).

Tension artérielle orthostatique. La dégradation du système nerveux périphérique altère le signalement des variations de la tension artérielle relatives à la position, entraînant une réponse différée ou atténuée du système nerveux sympathique aux changements de position. La mesure indirecte de la tension artérielle (TA) est une mesure clinique bien établie, dont la stabilité par rapport aux changements de position du corps est avérée, s’il n'existe pas de pathologie sous-jacente (Quaal, 1997). Les mesures de tension artérielle ont été effectuées avec un tensiomètre médical Vasotrac ${ }^{\circledR}$ de Medwave (Medwave, Inc. St. Paul, MN) électronique et calibré. Les mesures ont été prises en position couchée, assise et debout. Le Vasotrac ${ }^{\circledR}$ a un capteur non invasif, fixé au niveau de l'artère radiale par une bande Velcro, et l'information est alors lue à partir du pouls radial. La lecture de la tension artérielle systolique, diastolique et moyenne s'exprime en $\mathrm{mmHg}$. Les mesures de TA du Vasotrac ${ }^{\circledR}$ présentent une corrélation moyenne de 0,97 avec un cathéter intraartériel, offrant ainsi la précision nécessaire pour détecter les variations (Belani, Ozaki, Hynson, Hartmann, Reyford, Martino et al., 1999). Les variations de la tension artérielle orthostatique entre les positions couchée et debout ont été mesurées sur une échelle continue en $\mathrm{mmHg}$.

Force musculaire. La force musculaire des fléchisseurs et des extenseurs du genou et de la hanche a été mesurée à l'aide d'un dynamomètre manuel. Le goniomètre est placé au-dessus du genou et de la cheville, et une mesure est enregistrée pendant que le patient, en position de soutenir le poids de son corps, effectue une série de mouvements. Dans les études précédentes, la validité du goniomètre avait été établie par rapport à d'autres exercices exigeant un effort équivalent. Les coefficients de corrélation intra-test de la dynamométrie manuelle varient de 0,82 à 0,96 pour les sujets ayant une neuropathie préexistante et les sujets de contrôle (Bohannon et Andrews, 1987). Dans cette étude, la fiabilité intra-évaluateur du dynamomètre variait entre 0,88 et 0,90 .

Marche et équilibre. La marche a été évaluée selon le volet « marche » de l'instrument d'évaluation de la marche et de l'équilibre de Tinetti, axé sur la performance (Tinetti, 1986). Cette évaluation en 17 items de la mobilité exige du sujet l'exécution d'exercices faisant appel à la stabilité. La marche a été observée sous trois aspects : en marchant dans la direction de l'examinateur, en s'éloignant de ce dernier, et sur un plan perpendiculaire. Les items étaient notés 0 ou 1 , la note zéro étant considérée comme anormale, indiquant une incapacité de marcher ou la nécessité d'une assistance pour la marche. Une marche normale recevait la note 1. Les items de la marche comprenaient l'initiation de la marche, la hauteur du pas, la longueur du pas, la symétrie du pas, la continuité du pas, la déviation de la marche, la stabilité du tronc, la marche, l'aplomb et la manière de tourner en marchant. La somme des cotes des différents items fournissait le score de la marche, lequel s'échelonnait de 0 à 9 , où 9 équivalait à une marche normale.

L'équilibre dynamique a été évalué selon le volet « équilibre » de l'instrument de Tinetti (Tinetti, 1986), comprenant les items suivants : 
l'équilibre en position assise, se lever d'une chaise, l'équilibre immédiat debout, tourner la nuque, lever les bras, se baisser et s'asseoir. L'équilibre était noté de 0 à 2 . La note 0 était considérée comme anormale, indiquant l'incapacité de compenser les déficits en matière d'équilibre. La note 1 indiquait que le sujet avait une réponse normale avec une assistance et qu'il était capable d'utiliser les moyens compensateurs de maintien de l'équilibre. La note 2 représentait pour chaque item un équilibre normal. Les cotes étaient additionnées pour obtenir un score total, variant de 0 à 16 , les scores plus faibles indiquant une stabilité posturale moindre. La fidélité interjuges et le coefficient test-retest sont $>0,95$ (Spellbring et Ryan, 1997). Les indications cliniques de troubles de la marche et de l'équilibre méritent une attention particulière, car ces troubles se prêtent à des interventions susceptibles d'améliorer la santé fonctionnelle.

Souffrance liée aux symptômes. La souffrance liée aux symptômes a été mesurée selon l'échelle de perception des symptômes («SEI » selon son acronyme anglais) nouvellement révisée, dont les 34 items originaux ont été portés à 48. (Rhodes, McDaniel, Homan, Johnson et Madsen, 2000). La grille originale est une échelle d'autoévaluation de type Likert qui évalue 15 symptômes : nausée, vomissement, douleur, anorexie, sommeil, fatigue, élimination des selles, respiration, toux, concentration, image corporelle, larmoiement, température corporelle et nervosité. Les items sont classés en six souséchelles de perception des symptômes (respiratoire, douleur/gêne, fatigue/nervosité, gastro-intestinal, concentration et enfin, apparence) et notés de 0 (absence du symptôme) à 4 (grande fréquence). Cet instrument fournit des mesures sur la perception totale des symptômes, la fréquence des symptômes et la souffrance liée aux symptômes. L'alpha de Cronbach s'établissait à 0,91 pour la perception globale des symptômes, 0,76 pour la souffrance globale, et 0,90 pour la fréquence globale des symptômes. La fiabilité des sous-échelles variait de 0,64 à 0,84 (concentration), à l'exception de l'apparence à 0,38 . Les coefficients test-retest étaient très convergents $(r=0,92)$. La validité de l'instrument s'appuyait sur le test de Kruskal-Wallis et sur la validité du contenu, car il incluait les symptômes identifiés par les patients. L'instrument original avait été révisé en collaboration avec les Drs Rhodes et McDaniel, les auteurs de l'instrument, pour y ajouter les 14 items reflétant les symptômes associés à la neuropathie périphérique, telles que les sensations de brûlure, d'engourdissement et de fourmillement. Pour cette étude, les scores de l'alpha de Cronbach pour la sous-échelle de la neuropathie périphérique étaient de 0,66 au niveau de référence, de 0,62 à 4 semaines et de 0,79 à 12 semaines. La fiabilité des scores augmente avec la gravité et la fréquence des symptômes.

Tableau un : Sommaire des instruments et des scores

\begin{tabular}{|c|c|c|}
\hline Variable et mesure & Procédure & Étendue des scores \\
\hline $\begin{array}{l}\text { Vision } \\
\text { Échelle Snellen manuelle }\end{array}$ & $\begin{array}{l}\text { Le sujet lisait l'échelle tenue à } 14 \text { po, en } \\
\text { couvrant l'œil opposé. }\end{array}$ & $20 / 20-20 / 800$ \\
\hline $\begin{array}{l}\text { Audition } \\
\text { Épreuve de Weber } \\
\text { Épreuve de Rinne } \\
\text { La fourche du diapason excité } \\
\text { était placée sur le crâne, au } \\
\text { niveau du méridien. }\end{array}$ & $\begin{array}{l}\text { La fourche du diapason excité était placée } \\
\text { contre les apophyses mastoïdes (conduction } \\
\text { osseuse) jusqu'à ce que le sujet ne l'entende } \\
\text { plus. Le diapason était ensuite dirigé vers le } \\
\text { devant de l'oreille testée pour vérifier la } \\
\text { conduction aérienne. } \\
0 \text { si le son est perçu latéralement (anormal) } \\
1 \text { si le son est perçu symétriquement (normal) }\end{array}$ & $\begin{array}{l}0=\text { son non perçu } \\
1=\text { son perçu }\end{array}$ \\
\hline $\begin{array}{l}\text { Sensibilité cutanée } \\
\text { Test de sensibilité par les } \\
\text { monofilaments de Semmes- } \\
\text { Weinstein }\end{array}$ & $\begin{array}{l}\text { Filaments de nylon calibrés de diverses } \\
\text { épaisseurs appliqués à différentes zones des } \\
\text { mains et des pieds. }\end{array}$ & $\begin{array}{l}\text { Le filament est appliqué pendant } 3 \text { secondes sur des } \\
\text { zones spécifiques de chaque main ( } 6 \text { zones) et de chaque } \\
\text { pied ( } 4 \text { zones), pendant que le sujet garde les yeux } \\
\text { fermés. La note obtenue correspond au monofilament } \\
\text { ressenti, les valeurs supérieures indiquant une sensibilité } \\
\text { moindre au toucher. Cette méthode standardisée offre un } \\
\text { intervalle de confiance de } 95 \% \text {. Pour les mains, les } \\
\text { cotations allaient de } 1 \text { (filament le plus fin) à } 5 \text { (filament } \\
\text { le plus épais) pour chacune des six zones des mains } \\
\text { testées. Pour les pieds, les cotations allaient de } 1 \\
\text { (filament le plus fin) à } 3 \text { (filament le plus épais) pour } \\
\text { chacune des } 4 \text { zones des pieds testées. Le total des } \\
\text { cotations correspondant aux } 20 \text { zones testées va de } 20 \\
\text { (pouvoir de discrimination maximal) à } 96 \text { (pouvoir de } \\
\text { discrimination minimal). }\end{array}$ \\
\hline $\begin{array}{l}\text { Vibration } \\
\text { Diapason calibré à } 128 \mathrm{~Hz} .\end{array}$ & $\begin{array}{l}\text { Le diapason excité était appliqué sur les } \\
\text { parties latérales droite et gauche de chaque } \\
\text { genou et de chaque cheville. }\end{array}$ & $\begin{array}{l}\text { Les résultats étaient soit } 0=\text { incapacité à percevoir le } \\
\text { début ou la fin de la vibration soit } 1=\text { capacité à } \\
\text { percevoir le début ou la fin de la vibration. }\end{array}$ \\
\hline $\begin{array}{l}\text { Réflexes tendineux } \\
\text { Marteau à réflexes de Babinski }\end{array}$ & $\begin{array}{l}\text { Les réflexes ont été provoqués en tapotant le } \\
\text { tendon d'un muscle étiré selon des procédés } \\
\text { cliniques normalisés. }\end{array}$ & $\begin{array}{l}0=\text { absence de réflexe } \\
1=\text { trace de réflexe } \text { inférieure à la normale } \\
2=\text { réflexe situé dans la moitié inférieure de la plage } \\
\text { normale } \\
3=\text { réflexe situé dans la moitié supérieure de la plage } \\
\text { normale } \\
4=\text { réflexe prononcé }\end{array}$ \\
\hline
\end{tabular}




\section{Résultats}

Types de changements. Les changements de la fonction nerveuse périphérique sont présentés selon une approche de la tête aux pieds pour l'évaluation des variables liées aux résultats. Le graphique des types de changements de la fonction nerveuse périphérique se trouve dans le tableau 2. Le score moyen de l'acuité visuelle était de 20/86 au niveau de référence, et chutait à 20/57 à 4 semaines et à 20/39 à 12 semaines de thérapie. L'épreuve de Rinne n'a pas montré de changements notables dans la conduction aérienne et osseuse du son de l'appareil auditif. En revanche, l'épreuve de Weber a révélé des altérations dans la conduction osseuse du son. Au niveau de référence, $82 \%$ des participants étaient capables d'entendre un son de façon symétrique, une audition normale. Après 4 semaines de thérapie, $54 \%$ seulement des participants disaient entendre le son de façon symétrique, et ce taux était également obtenu lors de l'évaluation suivante à 12 semaines.

Le score moyen de la sensibilité cutanée pour les mains et les pieds était de 27 au niveau de référence, légèrement au-dessus du score considéré le plus sensible, mais encore normal. Le score moyen a grimpé à 30 à 4 semaines et à 32,5 à 12 semaines de traitement, indiquant un déclin de $6 \%$ de la sensibilité tactile. Le score moyen pour les mesures de la sensibilité vibratoire aux emplacements bilatéraux des genoux et des chevilles était de 7,6 au niveau de référence; il est resté stable à 4 semaines mais est tombé à 6,8 à 12 semaines, ce qui représente un écart de $10 \%$ par rapport au niveau de référence. Le score moyen pour les réflexes tendineux des extrémités inférieures était de 5,7 au niveau de référence, il est tombé à 4,6 (19\%) à 4 semaines, mais est remonté à 5,5 à 12 semaines pour un déclin global des réflexes de $4 \%$.

Le score moyen des variations de la tension artérielle orthostatique de la position couchée à la position debout était de $6,2 \mathrm{mmHg}$ au niveau de référence; il est monté à $13,4 \mathrm{mmHg}$ à la semaine 4 , avant de chuter à 3,1 - en dessous du niveau de référence - à la semaine 12 . Le score moyen de la marche au niveau de référence était de 9 , le score maximal. Ce score est resté stable pendant toute la durée de l'étude. Le score moyen de l'équilibre était de 15,5 au niveau de référence, tombant à 14,3 à la semaine 4 et demeurant stable à 12 semaines de traitement. Le score moyen de la force musculaire des extrémités inférieures était de 41,2 au niveau de référence, tombant à 39,5 à la semaine 4 et continuant de décliner à 37,1 à 12 semaines de traitement.

L'échelle de la perception des symptômes a mis en évidence la corrélation entre les symptômes de neuropathie auto-rapportés et la souffrance liée aux symptômes, tant à 4 qu'à 12 semaines de traitement (tableau 3). Les altérations de température corporelle auto-rapportées, comme la sensation de froid, entretenaient des liens étroits avec la souffrance liée aux symptômes à 4 semaines de traitement. Les symptômes classiques de neuropathie auto-rapportés (douleur, engourdissement, et fourmillement aux extrémités) entretenaient des rapports étroits avec la souffrance liée aux symptômes à 4 et à 12 semaines; il apparaît clairement que la souffrance liée aux symptômes augmentait lorsque des changements intervenaient dans les extrémités. Les désordres gastro-intestinaux, particulièrement la constipation, correspondaient à la souffrance liée aux symptômes à 4 semaines, mais s'étaient dissipés à 12 semaines. Les pertes auditives subjectives étaient corrélées négativement avec la souffrance liée aux symptômes à 4 semaines, mais pas à 12 semaines. Les variations orthostatiques de la tension artérielle observées à 4 semaines semblaient causer une souffrance, mais le problème s'était résolu à 12 semaines de thérapie. Les troubles de la marche auto-rapportés étaient corrélés négativement avec la souffrance liée aux symptômes à 12 semaines seulement. Le tableau 4 résume les changements intervenus dans la fonction nerveuse périphérique durant les 12 semaines de la période étudiée.

\section{Discussion}

Ce travail constitue la seule étude détaillée connue des effets neurotoxiques du traitement biothérapique du mélanome malin. De nombreux paramètres physiologiques examinés ici ne font pas partie de l'évaluation de la toxicité communément effectuée par les infirmières en oncologie, mais ont des implications pour une transposition dans la pratique clinique. Pour garantir la précision des résultats, les infirmières en oncologie effectuant des mesures cliniques d'évaluation de la toxicité, tels les tests des réflexes tendineux, de l'audition et de la

\begin{tabular}{|c|c|c|}
\hline \multicolumn{3}{|l|}{... suite de la page 170} \\
\hline $\begin{array}{l}\text { Tension artérielle orthostatique } \\
\text { Vasotrac }^{\circledR} \text { de Medwave }\end{array}$ & $\begin{array}{l}\text { Un capteur est placé sur l'artère radiale et } \\
\text { maintenu en place au moyen d'une bande } \\
\text { Velcro. Les valeurs de la tension systolique, } \\
\text { diastolique et moyenne sont prises. }\end{array}$ & La tension était mesurée en $\mathrm{mmHg}$. \\
\hline $\begin{array}{l}\text { Force musculaire } \\
\text { Dynamomètre manuel }\end{array}$ & $\begin{array}{l}\text { Le dynamomètre est placé au-dessus du genou } \\
\text { et de la cheville, et une mesure est enregistrée } \\
\text { pendant que le patient, en position de soutenir } \\
\text { le poids de son corps, effectue une série de } \\
\text { mouvements. }\end{array}$ & La force maximale est mesurée en $\mathrm{kg}$. \\
\hline $\begin{array}{l}\text { Marche : Instrument d'evaluation } \\
\text { de l'equilibre et de la marche de } \\
\text { Tinetti, axé sur la performance } \\
\text { (Tinetti, 1986). }\end{array}$ & $\begin{array}{l}\text { La marche était observée et notée en fonction } \\
\text { de trois aspects: en marchant dans la direction } \\
\text { de l'examinateur, en s'éloignant de ce dernier, } \\
\text { et sur un plan perpendiculaire. }\end{array}$ & $\begin{array}{l}0 \text { (marche anormale) } \\
1 \text { (marche normale) }\end{array}$ \\
\hline $\begin{array}{l}\text { Équilibre : Instrument } \\
\text { d'évaluation de l'equilibre et de } \\
\text { la marche de Tinetti, axé sur la } \\
\text { performance (Tinetti, 1986). }\end{array}$ & $\begin{array}{l}\text { Les exercices d'équilibre réalisés comprenaient : } \\
\text { équilibre en position assise, se lever d'une } \\
\text { chaise, équilibre immédiat debout, tourner la } \\
\text { nuque, lever les bras, se baisser et s'asseoir. }\end{array}$ & $\begin{array}{l}0=\text { équilibre anormal } \\
1=\text { équilibre avec compensation } \\
3=\text { équilibre normal }\end{array}$ \\
\hline $\begin{array}{l}\text { Souffrance liée aux } \\
\text { symptômes - Échelle de } \\
\text { perception des symptômes } \\
\text { (SEI) (Rhodes et McDaniel } \\
\text { et al, 2000). }\end{array}$ & $\begin{array}{l}\text { La SEI est une échelle ordinale de type Likert } \\
\text { de } 48 \text { items qui sert à l'autoévaluation de } 15 \\
\text { symptômes : nausée, vomissements, douleur, } \\
\text { anorexie, sommeil, fatigue, élimination des } \\
\text { selles, respiration, toux, concentration, image } \\
\text { corporelle, larmoiement, température } \\
\text { corporelle et nervosité. }\end{array}$ & $\begin{array}{l}\text { Cet instrument avait des cotations allant de } 0 \text { (aucune } \\
\text { survenue) à } 4 \text { (survenue la plus fréquente) et } \\
\text { fournissait des mesures de la perception globale des } \\
\text { symptômes, de la survenue des symptômes et de la } \\
\text { souffrance liée aux symptômes. }\end{array}$ \\
\hline
\end{tabular}


vision, doivent être compétentes dans la saisie et l'interprétation de ces mesures. Lors de l'utilisation de mesures cliniques pour la recherche, les problèmes de mesure doivent être pris en compte. On constate un besoin de méthodes plus précises d'évaluation de la vision et de l'audition en milieu clinique. Par exemple, cette étude utilisait une échelle d'acuité manuelle pour les tests visuels, comme la solution la plus pratique dans un service d'oncologie déjà fort occupé. Cependant, cette approche augmente la marge d'erreur de mesure, puisque le sujet peut manipuler la distance à laquelle il tient la carte en la rapprochant de son champ visuel par rapport aux recommandations du test, ce qui fausse les résultats. Dans cette étude, l'acuité visuelle affichait une tendance à l'amélioration, un résultat contredit par les rapports de cas publiés à propos des effets de l'interféron alpha sur la vision. De même, les tests de la conduction aérienne et de la conduction osseuse de l'appareil auditif, réalisés à l'aide d'un diapason, semblaient avoir une capacité limitée à détecter les troubles de l'audition. Les résultats de l'épreuve de Weber effectuée dans cette étude suggéraient que l'audition était affectée par l'interféron alpha pendant la phase du traitement comportant l'administration de doses intensives, alors que l'épreuve de Rinne ne montrait aucun changement de la conduction aérienne et de la conduction osseuse de l'appareil auditif par rapport au niveau de référence. Pour l'évaluation clinique de la toxicité de l'interféron alpha sur l'appareil auditif, on notera que l'épreuve de Weber permet une évaluation plus précise de l'audition et est au demeurant très facile à exécuter au chevet des patients. Cependant, l'utilisation d'un chronomètre pour enregistrer la durée pendant laquelle le son est perçu et d'une série de diapasons, particulièrement ceux ayant des fréquences plus élevées, pourrait augmenter la sensibilité de l'épreuve de Rinne en milieu clinique.

Les résultats de cette étude suggèrent qu'il y a des déclins de la sensibilité cutanée, de la sensibilité vibratoire et de la force musculaire liés aux doses, qui se produisent en conjonction avec des régimes à haute dose d'interféron alpha. Du fait du caractère expérimental de cette étude et du temps de suivi relativement court, on ne sait pas précisément si ces déclins persistent, s'aggravent ou
Tableau deux : Caractéristiques de la fonction nerveuse périphérique
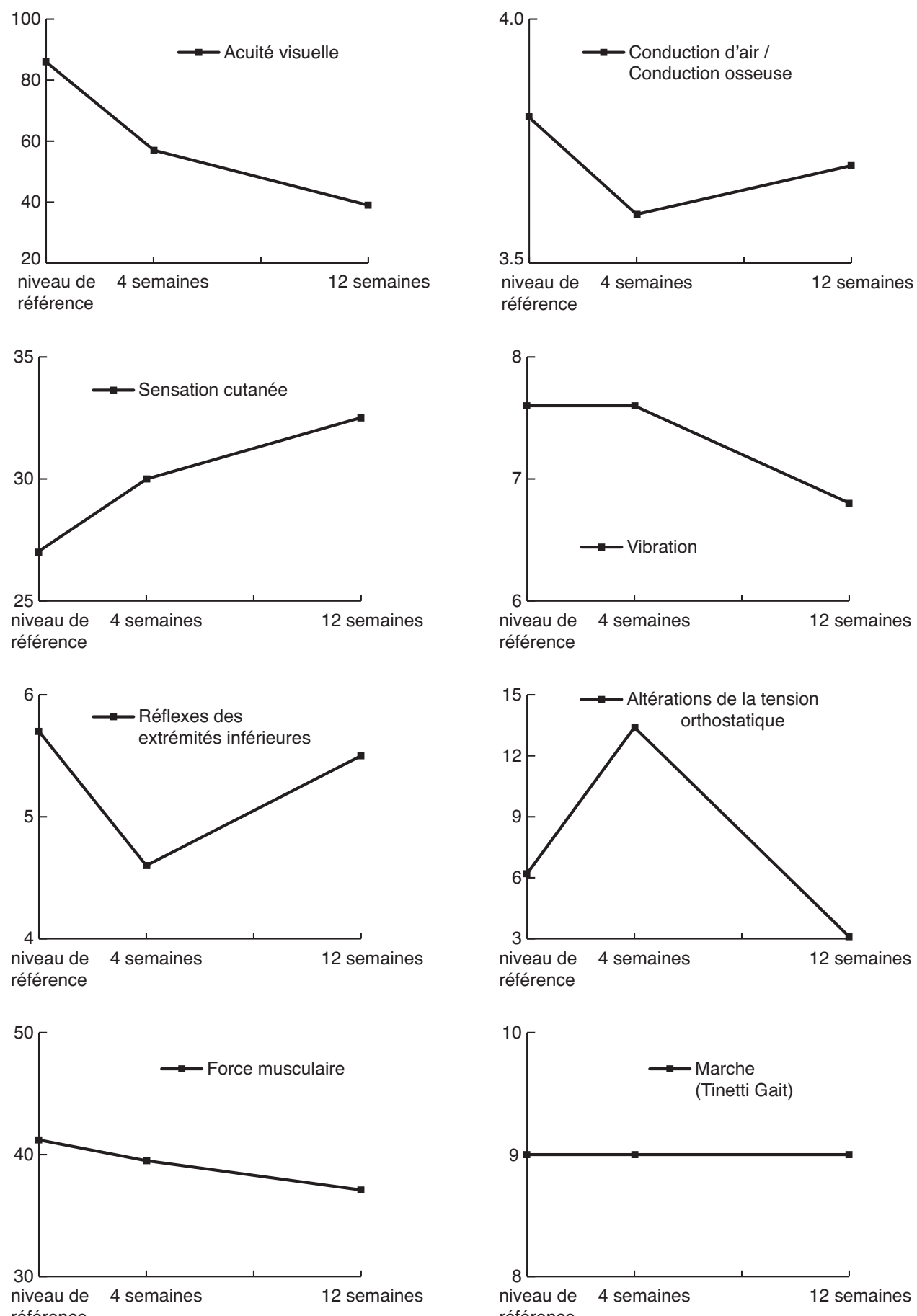

référence
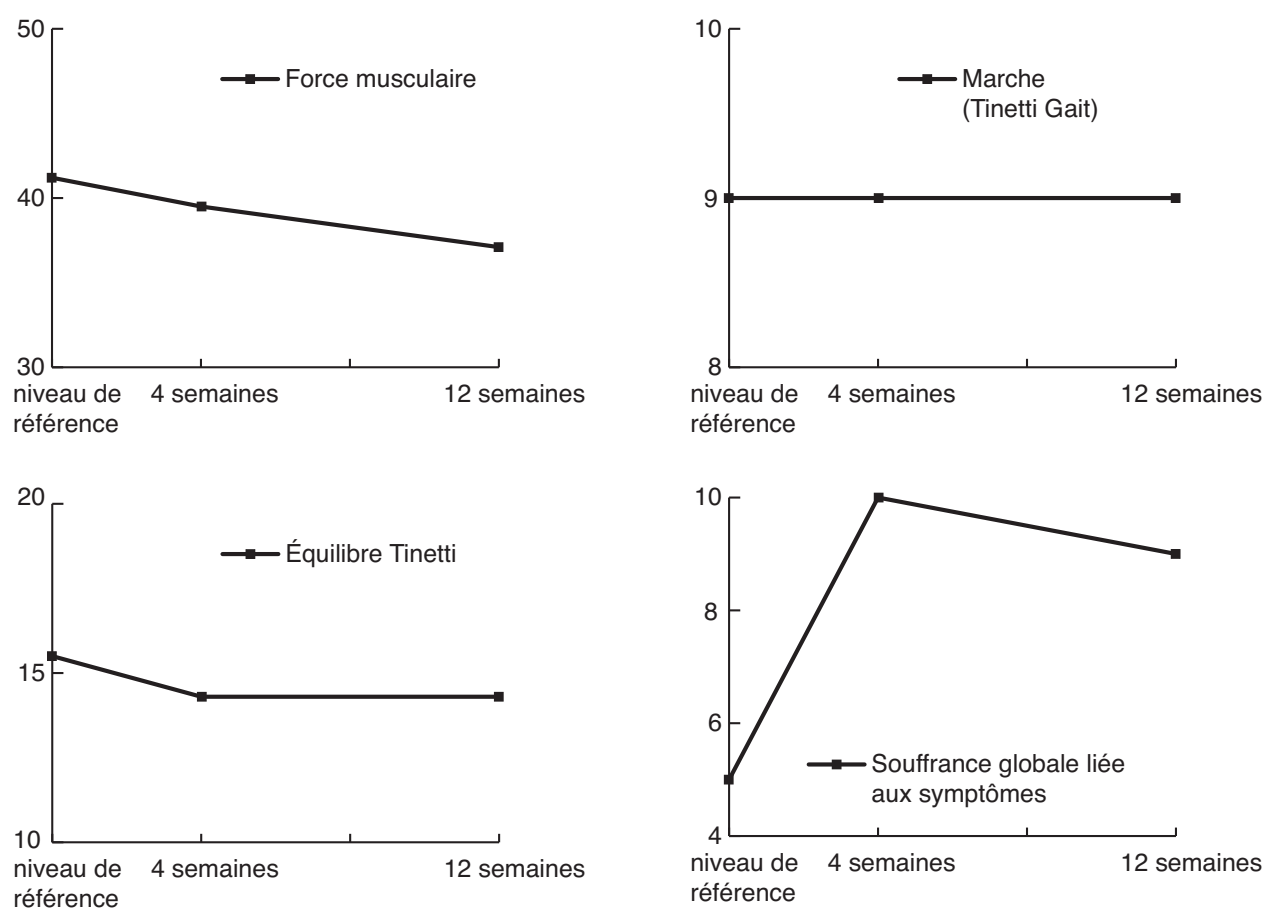
se résolvent sur la durée normale d'un an du régime de traitement à l'interféron alpha. Les réflexes tendineux dans les extrémités inférieures étaient inchangés tout au long de la période étudiée. L'analyse des réflexes tendineux est très subjective et comporte des difficultés d'interprétation, même pour des infirmières cliniciennes expérimentées.

Des altérations de la tension artérielle liée aux changements de position ont été notées pendant les 4 premières semaines de traitement, durant lesquelles le sujet se trouvait plus exposé à des blessures par chute. Ces altérations sont peut-être dues à des mécanismes cardiovasculaires autonomiques affectant le contrôle sympathique de la pression sanguine. Ces résultats soulignent la nécessité pour les infirmières en oncologie de surveiller les paramètres de la tension artérielle orthostatique tout au long du premier mois de thérapie. Il est possible qu' au cours des premières semaines de traitement, les patients ressentent des symptômes tels que la nausée, les vomissements, la diminution de l'appétit et de la prise de liquides qui contribuent à la déperdition volémique et exposent davantage les patients à des variations de la tension artérielle orthostatique. Les évaluations infirmières régulières du poids, de l'ingesta et de l'excreta, du pli cutané et des muqueuses, fourniraient des informations sur l'état du volume liquidien des patients pendant cette période critique. Après le premier mois de traitement, les facteurs responsables de la déplétion du volume liquidien auront probablement été pris en charge par les cliniciens. Les patients présentant des symptômes d'étourdissement ou de vertige devraient être instruits par les infirmières en oncologie sur les adaptations comportementales envisageables, comme se lever lentement pour faciliter l'ajustement physiologique de la pression sanguine, particulièrement pendant la phase de traitement à dose intensive. Les rapports subjectifs de symptômes associés avec les changements orthostatiques ne font pas partie de la surveillance de routine pour cette population, et des recherches supplémentaires sont nécessaires pour mieux comprendre ce phénomène.

Les scores de la marche et de l'équilibre, mesurés avec l'instrument d'évaluation de la marche et de l'équilibre de Tinetti axé sur la performance (Tinetti, 1986), étaient largement inchangés durant la période de l'étude. Chaque participant avait atteint le degré maximal (9) de l'instrument au niveau de référence, ce qui montre le niveau de performance relativement élevé des patients retenus pour un traitement du cancer en clinique externe. Si aucun changement de score dans la marche n'a été observé, la période d'étude n'a peut-être pas été suffisamment longue pour saisir les effets de la thérapie neurotoxique sur les paramètres de la marche. D'autres études, incluant la marche comme variable, s'appuyaient sur l'évaluation du chercheur de la

Tableau trois : Corrélations entre les symptômes de la neuropathie périphérique et la souffrance liée aux symptômes

\begin{tabular}{|l|l|l|}
\hline Souffrance liée aux symptômes & À 4 semaines & À 12 semaines \\
\hline $\begin{array}{l}\text { Ensemble des symptômes de } \\
\text { la neuropathie périphérique }\end{array}$ & 0,76 & 0,76 \\
\hline $\begin{array}{l}\text { Changements de la } \\
\text { température du corps }\end{array}$ & 0,88 & $\begin{array}{l}\text { Aucune } \\
\text { corrélation }\end{array}$ \\
\hline $\begin{array}{l}\text { Douleur, engourdissement, } \\
\text { sensation de fourmillement } \\
\text { aux extrémités }\end{array}$ & 0,91 & 0,88 \\
\hline $\begin{array}{l}\text { Troubles GI } \\
\text { Acuité auditive }\end{array}$ & 0,71 & $\begin{array}{l}\text { Aucune } \\
\text { corrélation }\end{array}$ \\
\hline $\begin{array}{l}\text { Altérations de la tension } \\
\text { artérielle orthostatique }\end{array}$ & 0,73 & $\begin{array}{l}\text { Aucune } \\
\text { corrélation }\end{array}$ \\
\hline
\end{tabular}

mobilité observée et l'examen du signe de Romberg, ou sur les troubles de la marche relatés par le patient (Postma, Hoekman, van Riel, Heimans et Vermorken, 1999). Aucune étude antérieure ne contenant une évaluation systématique de la marche au moyen de l'instrument de Tinetti, les comparaisons entre les études n'étaient pas possibles. L'équilibre a décliné de $5 \%$ par rapport au niveau de référence sur la durée du traitement. L'équilibre n'a pas été évalué dans les études examinant les effets de la biothérapie et de nouvelles évaluations sont nécessaires, particulièrement chez les sujets plus âgés, qui peuvent présenter des dégradations de la force musculaire et de la fonction nerveuse périphérique, liées à l'âge ou à la maladie. Les sujets recevant de ortes doses d'interféron alpha peuvent avoir besoin d'interventions éducatives et/ou d'appareils fonctionnels pour se mouvoir sans danger; par conséquent, une orientation pertinente vers des services de physiothérapie pour l'évaluation de l'équilibre et de la marche devrait être entreprise si l'équilibre s'avérait déficient.

Cette étude a utilisé l'échelle de perception des symptômes (SEI) nouvellement révisée pour mesurer la souffrance liée aux symptômes de la neuropathie périphérique. La souffrance accompagnant les sensations de froid et la constipation peut être le fait d'une détérioration de la réactivité du système nerveux autonomique pendant la thérapie à dose intensive de 4 semaines. Des rapports subjectifs de pertes auditives du niveau de référence à 4 semaines de traitement peuvent représenter un phénomène à court terme, associé avec l'administration intraveineuse de fortes doses d'interféron alpha, qui justifierait des recherches complémentaires. Des troubles de la marche auto-rapportés ont été notés à 12 semaines de thérapie, indiquant que des altérations de la marche/de l'équilibre, nécessitant parfois des interventions infirmières pour prévenir les chutes, pourraient survenir plus tard dans la phase de traitement.

La valeur de cette étude est limitée, en raison du petit échantillon de convenance utilisé et du manque de diversité raciale. Toutefois, il faut signaler que la majorité des individus qui

Tableau quatre

Altérations de la fonction nerveuse périphérique

\begin{tabular}{|l|l|l|l|}
\hline Mesures & $\begin{array}{l}\text { Niveau de } \\
\text { référence } \\
\text { X (É-T) }\end{array}$ & $\begin{array}{l}\text { 4 semaines } \\
\text { X́́-T) }\end{array}$ & $\begin{array}{l}\text { 12 semaines } \\
\text { (́́-T) }\end{array}$ \\
\hline Acuité visuelle & $20 / 86(76)$ & $20 / 56(59)$ & $20 / 39(16)$ \\
\hline $\begin{array}{l}\text { Audition } \\
\text { Épreuve de Rinne } \\
\text { Weber - } \\
\text { symétriquement } \\
\begin{array}{l}\text { Weber - } \\
\text { latéralement }\end{array}\end{array}$ & $\begin{array}{l}3,8(0,40) \\
9(82 \%)\end{array}$ & $\begin{array}{l}3,5(0,52) \\
6(54,5 \%)\end{array}$ & $\begin{array}{l}3,7(0,75) \\
6(54,5 \%)\end{array}$ \\
\hline Sensibilité cutanée & $27(8,4)$ & $30(8,7)$ & $32(10,4)$ \\
\hline Vibration & $7,6(1,2)$ & $7,6(0,70)$ & $6,8(1,6)$ \\
\hline $\begin{array}{l}\text { Réflexes tendineux } \\
\text { des extrémités } \\
\text { inférieures }\end{array}$ & $5,8(2,2)$ & $4,7(2,5)$ & $5,4(2,8)$ \\
\hline $\begin{array}{l}\text { Tension } \\
\text { orthostatique }\end{array}$ & $6,2(13,5)$ & $13,4(13,0)$ & $3,1(8,6)$ \\
\hline Force musculaire & $41,2(9,0)$ & $39,5(9,1)$ & $37,1(6,4)$ \\
\hline Marche & 9,0 & 9,0 & 8,8 \\
\hline Équilibre & 15,5 & 14,3 & 14,7 \\
\hline $\begin{array}{l}\text { Souffrance liée } \\
\text { aux symptômes }\end{array}$ & 4,2 & 9,7 & 9,1 \\
\hline
\end{tabular}


développent un mélanome malin sont des Blancs à la peau claire, ainsi que le reflète l'échantillon en question. Cette étude montre la nécessité d'une surveillance neurologique conséquente et permanente des patients recevant des agents biothérapeutiques. Les troubles de la marche et de l'équilibre n'ont peut-être pas été observés au début du traitement, mais puisque les patients ont fait état de tels symptômes après 12 semaines de traitement, une évaluation de la marche et de l'équilibre devrait être poursuivie tout au long de la thérapie.

En résumé, il y a des changements de la sensibilité cutanée, de la sensibilité vibratoire, de la force musculaire et de la tension artérielle dans les 12 premières semaines d'administration de fortes doses d'interféron alpha-2b. Bien que l'utilisation de mesures cliniques pour évaluer l'apparition et la gravité de la neuropathie soit à la fois économique et facile à mettre en œuvre dans cette population, leur emploi pour la surveillance de la toxicité clinique ou la recherche suscite des défis. Les mesures cliniques d'usage courant peuvent ne

\section{Références}

Armstrong, T., Rust, D., \& Kohtz, J. (1997). Neurologic, pulmonary and cutaneous toxicities of high-dose chemotherapy. Oncology Nursing Forum Supplement, 24(1), 23-33.

Belani, K., Ozaki, M., Hynson, J., Hartmann, T., Reyford, H., Martino, J.M., Poliac, M., \& Miller, R. (1999). A new noninvasive method to measure blood pressure: Results of a multicenter trial. Anesthesiology, 91(3), 686-692.

Berger, T., Malayeri, R., Doippelbauer, A., Krajnik, G., Huber, H., Auff, E., \& Pirker, R. (1997). Neurological monitoring of neurotoxicity induced by paclitaxel/cisplatin chemotherapy. European Journal of Cancer, 33(9), 1393-1399.

Birke, J., \& Sims, D. (1985, December 16-18). Plantar sensory threshold in the Hansen's disease ulcerated foot. Proceedings of the International Conference on Biomechanics and Clinical Kinesiology of Hand and Foot, Madras, India.

Bohannon, R., \& Andrews, A. (1987). Interrater reliability of handheld dynamometry. Physical Therapy, 67, 931-933.

Bouldin, T. (1994). The peripheral nervous system. In Rubin \& Farber (Eds.), Pathology (pp 1447-1455). Philadelphia: J.B. Lippincott Company.

Bradley, A., Hook, J., \& Haeseker, J. (1991). A comparison of clinical acuity and contrast sensitivity charts: effect of uncorrected myopia. Opthalmic Physiology, 11(3), 218-226.

Fattovich, G., Giustina, G., Favarato, S., Ruol, A., et al. (1996). A survey of adverse events in 11,241 patients with chronic viral hepatitis treated with alpha interferon. Journal of Hepatology, 24, 38-47.

Gastineau, D., Habermann, T., \& Hermann, R. (1989). Severe neuropathy associated with low-dose recombinant interferonalpha. American Journal of Medicine, 87,116.

Gotaskie, G., \& Andreassi, B. (1994). Paclitaxel: A new antimitotic chemotherapeutic agent. Cancer Practice, 2(4), 27-33.

Haapaniemi, J.J., Suonpaa, J.T, Salmivalli, A.J., \& Virolainen, E.S. (1996). C1-tuning fork tests in school-aged children. Auris Nasus Larynx, 23, 26-32.

Irwin, M. (1987). Patients receiving biologic response modifiers: Overview of nursing care. Oncology Nursing Forum, 14(6), 3237.

Klima, R., Weigand, A., \& DeLisa, J. (1991). Nerve conduction studies and vibration perception thresholds in diabetic and uremic neuropathy. American Journal of Physical and Medical Rehabilitation, 70(2), 86.

Manesis, E., Petrou, C., Brouzas, D., \& Hadziyannis, S. (1994). Optic tract neuropathy complicating low-dose interferon treatment. Journal of Hepatology, 21, 474-477. pas être suffisamment efficaces pour détecter les dysfonctionnements neurologiques précoces. Non seulement la saisie des mesures cliniques requiert un examinateur extrêmement exercé pour être conduite avec toute la constance et la précision nécessaires, mais la mesure particulière choisie et la nature subjective de certaines mesures limitent l'exactitude dans l'interprétation des résultats obtenus. Les études prospectives du suivi à long terme de la neuropathie périphérique en tant que complication du traitement du cancer sont peu nombreuses, et se concentrent largement sur l'observation des symptômes durant les essais pharmacologiques. L'évaluation de la neuropathie périphérique en conjonction avec le cancer et les traitements du cancer n'est toujours pas normalisé. Une évaluation infirmière complète et normalisée améliorera le dépistage de la neuropathie induite par le traitement et pourrait fournir un modèle pour la surveillance neurologique des patients qui présentent un risque de développer une neuropathie périphérique induite par le traitement.

Merriggioli, M., \& Brown, J. (2000). Chronic inflammatory demyelinating polyneuropathy after treatment with interferonalpha. Muscle \& Nerve, 23, 433-435.

Meyers, C., Scheibel, R., \& Forman, A. (1991). Persistent neurotoxicity of systematically administered Interferon-Alpha. Neurology, 41, 672-676.

Miltenburg, D.M. (1994). The validity of tuning fork tests in diagnosing hearing loss. Journal of Otolaryngology, 23(4), 254-259.

Postma, T., Hoekman, K., van Riel, J.M.G.H., Heimans, J.J., \& Vermorken, J.B. (1999). Peripheral neuropathy due to biweekly paclitaxel, epirubicin and cisplatin in patients with advanced ovarian cancer. Journal of Neuro-Oncology, 45, 241-246.

Postma, T., \& Heimans, J. (2000). Grading of chemotherapy-induced peripheral neuropathy. Annals of Oncology, 11, 509-513.

Quaal, S. (1997). Measuring cardiac parameters. In Frank-Stromborg \& Olsen (Eds.), Instruments for clinical health-care research (pp. 445-458).

Quattrini, A., Comi, G., Nemni, R., Martinelli, V., Villa, A., Caimi, et al. (1997). Axonal neuropathy associated with interferon-alpha treatment for hepatitis C: HLA-DR immunoreactivity in Schwann cells. Acta Neuropathologica, 94, 504-508.

Rendell, M., Katims, J., \& Richter, R. (1989). A comparison of nerve conduction velocities and current perception thresholds as correlates of clinical severity of diabetic sensory neuropathy. Journal of Neurology and Neurosurgical Psychiatry, 52, 502.

Rhodes, V., McDaniel, R., Homan, S.H., Johnson, M., \& Madsen, R. (2000). An instrument to measure symptom experience. Cancer Nursing, 23(1), 49-54.

Smith, J., Longo, D., Urba, W., Clark, J., Watson, T., Beveridge, J., et al. (1991). Prolonged, continuous treatment of hairy cell leukemia patients with recombinant interferon-alpha 2A. Blood, 78(7), 1664-1671.

Spellbring, A., \& Ryan, J. (1997). Measuring mobility and potential for falls. In Frank-Stromborg \& Olsen (Eds.), Instruments for health-care research (pp.497-508).

Tinetti, M. (1986). Performance-oriented assessment of mobility problems in elderly patients. Journal of the American Geriatric Society, 34, 119-126.

Vial, T., \& Descotes, J. (1994). Clinical toxicity of the interferons. Drug Safety, 10(2), 115-150.

Visovsky, C. (2003). Chemotherapy-induced peripheral neuropathy. Cancer Investigation, 21(3), 439-451.

Wilkes, G. (1996). Neurological disturbances. In Groenwald, Frogge, Goodman \& Yarbro (Eds.), Cancer symptom management (pp. 324-355). Boston: Jones \& Bartlett. 